points are distributed along lines that represent the expected differences in signal intensity from each channel. Without a systematic dye effect or after proper normalization, we would see three sets of data points along three equally spaced lines parallel with slope one. On the other hand, the direct comparison of measurements for each dye on different slides can be interpreted with no normalization because our experimental setting does not require across-slide normalization. Therefore, the most direct way to assess the efficiency of RNA amplification and Cy3 and Cy 5 dye incorporation during RNA labeling is to directly compare measured quantities for each channel on pairs of slides. Figure $2 \mathrm{C}$ illustrates the results of these comparisons. For each channel, we show the estimated slope for the corresponding intensity comparison, which corresponds precisely to the expected values.

This procedure can be used to monitor the quality of targets after the very last step of the labeling protocol, without compromising the amount of labeled target available for hybridization. Importantly, other commercially available products can efficiently control for amplification but cannot determine the efficiency of the labeling reaction because this would require laser excitation or equivalent wavelengths. Furthermore, one can determine label efficiency using data (Figure 1C) to correct for the specific activity of labeled targets to facilitate data normalization. In the case of greater differences in labeling efficiency, one can repeat the labeling step prior to hybridization, thus avoiding data of lower quality that could impose potential distortions during normalization and eliminating the costs of hybridization.

\section{REFERENCES}

1.Baugh, L.R., A.A. Hill, E.L. Brown, and C.P. Hunter. 2001. Quantitative analysis of mRNA amplification by in vitro transcription. Nucleic Acids Res. 29:E29.

2.Feldman, A.L., N.G. Costouros, E. Wang, M. Qian, F.M. Marincola, H.R. Alexander, and S.K. Libutti. 2002. Advantages of mRNA amplification for microarray analysis. BioTechniques 33:906-914.

3.Wang, E., L.D. Miller, G.A. Ohnmacht, E.T. Liu, and F.M. Marincola. 2000. High-fidel- ity mRNA amplification for gene profiling. Nat. Biotechnol. 18:457-459.

4.Quackenbush, J. 2002. Microarray data normalization and transformation. Nat. Genet. 32(Suppl)496-501.

5.Yang, Y.H., S. Dudoit, P. Luu, D.M. Lin, V. Peng, J. Ngai, and T.P. Speed. 2002. Normalization for cDNA microarray data: a robust composite method addressing single and multiple slide systematic variation. Nucleic Acids Res. 30:E15.
Received 12 May 2003; accepted 8 July 2003.

Address correspondence to Luiz F.L. Reis, Ludwig Institute for Cancer Research and Hospital do Câncer A.C. Camargo, Rua Prof. Antonio Prudente 109, 4th floor, São Paulo, SP, Brazil, 01509-010. e-mail: lreis@ludwig.org.br

\title{
Counter-selection facilitated plasmid construction by homologous recombination in Saccharomyces cerevisiae
}

\author{
Peter Robert Anderson ${ }^{1}$ and Yousef Haj-Ahmad ${ }^{2}$ \\ ${ }^{1}$ University of Guelph, Guelph and ${ }^{2}$ Brock University, St. Catharines, ON, Canada
}

BioTechniques 35:692-698 (October 2003)

The ability to shuffle and combine separate DNA fragments is one of the most fundamental activities in molecular biology. For this reason, in vitro manipulation of bacterial plasmid DNA using restriction enzymes is a staple technique of virtually every molecular biologist. Unfortunately, the position and availability of unique or rare restriction enzyme recognition sequences typically dictate the entire direction of subcloning strategies.

Recombinational cloning techniques based on homologous recombination in Saccharomyces cerevisiae can be used to circumvent tedious multiple-step plasmid construction strategies without the need for specific restriction sites (1-4). Unfortunately, the time investment and additional workload associated with the isolation and characterization of the desired recombinant plasmid from heterogeneous populations of S. cerevisiae transformants is a limitation of these otherwise valuable techniques.

The screening workload associated with in vivo plasmid construction techniques in S. cerevisiae has been partially abrogated by the use of various counter-selection techniques to isolate yeast transformants containing only recombinant plasmid $(1,3)$. However, these useful systems have, to this point, been limited exclusively to the construction of specific yeast expression vectors or required time-consuming replica plating to distinguish yeast transformants carrying recombinant plasmid. Here we describe a general method for URA3-based counter-selection during recombinational cloning that effectively eliminates the growth of background yeast transformants carrying regenerated acceptor plasmid by selecting for the growth of yeast containing only recombinant plasmid. Positive yeast transformants generated using this technique harbor exclusively the recombinant plasmid of interest without the need for replica plating (Figure 1).

The utility of the URA3-based counter-selection technique was demonstrated during in vivo construction of adenovirus E1-deletion rescue plasmids (used for the production of recombinant adenovirus vectors). The in vitro manipulation of such viral genomes as bacterial plasmids is typically difficult due to their large size and general lack of unique or rare restriction sites within the virus coding regions being manipulated. An adenovirus rescue vector capable of undergoing recombinational 
cloning in S. cerevisiae (YRpXC38) was generated by inserting the 1456-bp TRPI/ARS1 (chromosomal origin of replication) EcoRI fragment from YRp7 (5) into the unique EcoRI site of pXC38 (6). In addition to the appropriate yeast and bacterial replication origins and selectable markers, this vector contains nucleotides 22 to 5788 from the left end of the human adenovirus type 5 (Ad5) $\left(\right.$ GenBank $^{\circledR}$ accession no. X02996). URA3-based counter-selection of this plasmid during recombinational cloning was then made possible by embedding the $S$. cerevisiae URA3 gene within the region of the acceptor molecule that would be deleted during a successful recombinational cloning reaction. Briefly, PCR amplification of the URA3 gene from the plasmid pYES2 (Invitrogen, Carlsbad, CA, USA) was carried out with primers containing $5^{\prime}$ overhangs encoding $K p n I$ recognition sequences (forward primer 5'-GGGGGTACCGCTTTTCAATTCAATTCATC-3'; reverse primer 5'-GGGGGTACCGGGTAATAACTGATATAATTA-3', KpnI sites indicated by underline). This PCR fragment was subsequently purified from agarose gels using the GENECLEAN ${ }^{\circledR}$ DNA isolation kit (Qbiogene, Carlsbad, CA, USA), digested with KpnI, and inserted into the adenovirus sequences within YRpXC38 at a unique KpnI site, producing the plasmid YRpETE. Electroporation of electrocompetent S. cerevisiae with YRpETE generated yeast colonies capable of growth on trp/ura ${ }^{-}$synthetic complete medium, but incapable of growth on $\operatorname{trp}^{-}$synthetic complete medium fortified with $0.1 \%$ 5-fluoroorotic acid (5-FOA) (7).

Recombinant adenovirus vectors are often used as mammalian expression vectors. Consequently, PCR-

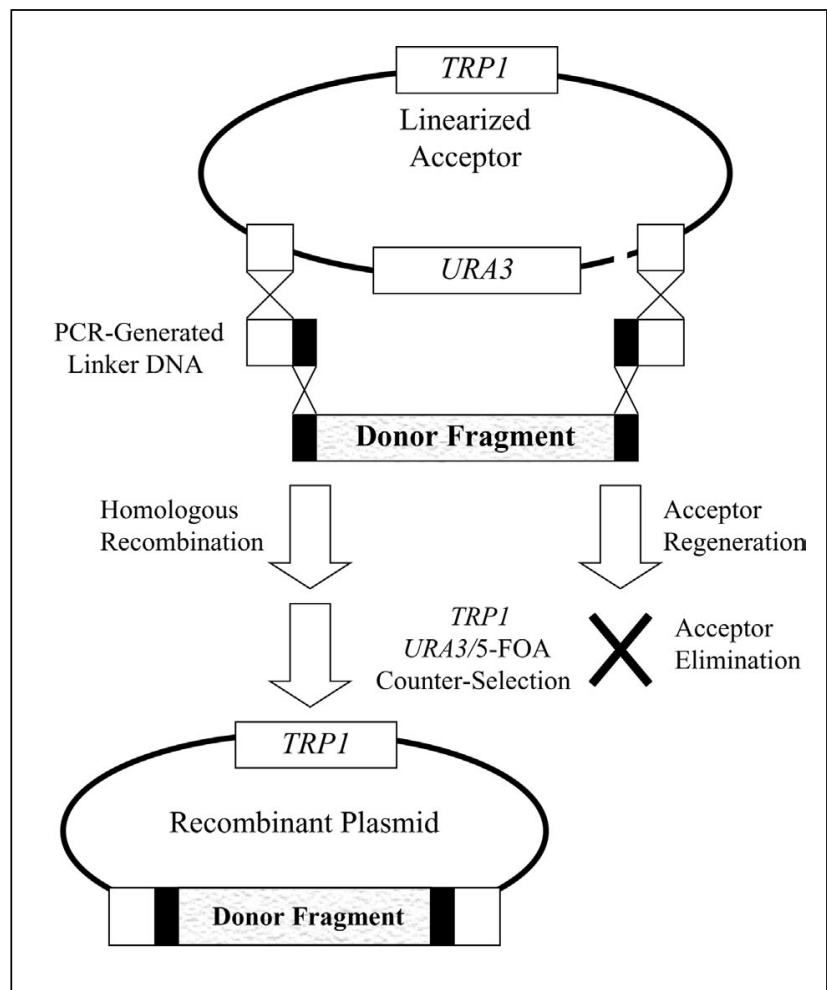

Figure 1. URA3-based counter-selection facilitated recombinational cloning strategy. A Saccharomyces cerevisiae URA3 selectable marker is inserted into the acceptor plasmid within a region that will be deleted during a successful recombinational cloning reaction. Subsequent deletion of the URA3 selectable marker resulting from homologous recombination in $S$. cerevisiae allows transformants harboring recombinant plasmid to survive $U R A 3 / 5$-fluoroorotic acid (5-FOA) counter-selection, while cells harboring the parental acceptor plasmid containing the URA3 marker (generated by resealing of cohesive ends within the acceptor plasmid) do not survive. derived mutations to any gene being inserted into the adenovirus backbone are undesirable. Linker-assisted homologous recombination $(1,4)$ was utilized to eliminate the need to PCR amplify the donor fragment. The relative positions of the regions that underwent homologous recombination and the reaction conditions and sequences of the oligonucleotides used to generate the linkers are depicted in Figure 2.

The donor fragment [a 1697-bp AflII/ AflIII fragment of pEGFP-N1 containing the enhanced green fluorescent protein (EGFP) reporter gene] was purified from agarose gels and resuspended at a final concentration of $1 \mu \mathrm{g} / \mu \mathrm{L}$. Typically, several isolations had to be pooled and concentrated by ethanol precipitation in order to obtain a donor fragment solution of sufficient concentration.

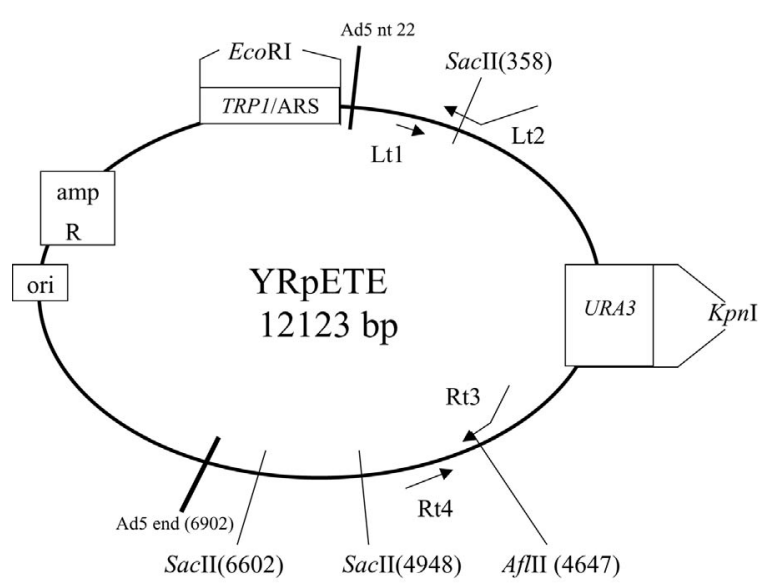

$$
\begin{aligned}
& \text { Lt1: 5'-cgacggatgtggcaaaag-3' } \\
& \text { Lt2: 5'-atgaactaatgaccccgtaattgattact } \\
& \text { attaataactagtctccacgtaaacggtc- } 3^{\prime} \\
& \text { Rt 3: 5'-aaatttcacaaataaagcattttttcact } \\
& \text { gcattctagtctgaaatgtgtgggcgtg-3' } \\
& \text { Rt 4: 5'-tcgtaggtcaaggtagtag-3' }
\end{aligned}
$$

Figure 2. Production of double-stranded linkers and positions of linearizing restriction sites in the YRPETE acceptor. PCR amplification of the linkers was carried out using strategies described previously (1). Oligonucleotides Lt1 and Lt2 were used to prime PCR amplification of bases 152 to 384 of the Ad5 genome from YRpETE. Oligonucleotides Rt 3 and Rt 4 were used to prime PCR amplification of bases 3514 to 3757 of the Ad5 genome from YRpETE. Lt2 and Rt3 each contain 40-base $5^{\prime}$ overhangs (nucleotide sequences in boldface) possessing homology to the first $40 \mathrm{bp}$ of the cytomegalovirus (CMV) promoter and the $3^{\prime}$ end of the green fluorescent protein (GFP) mRNA in pEGFP-N1 (BD Biosciences Clontech, Palo Alto, CA, USA), respectively. PCRs were carried out in $50-\mu \mathrm{L}$ reaction volumes containing $50 \mathrm{pmol}$ of both forward and reverse primers, 2.5 U of Taq DNA polymerase (New England Biolabs, Mississauga, ON, Canada), and the appropriate concentration of the accompanying buffers for 35 cycles of denaturation at $95^{\circ} \mathrm{C}$ for $1 \mathrm{~min}$, primer annealing at $47^{\circ} \mathrm{C}$ for $1 \mathrm{~min}$, and extension at $72^{\circ} \mathrm{C}$ for $1 \mathrm{~min}$. Reaction products were examined by agarose gel electrophoresis followed by ethanol precipitation and resuspension in Tris-EDTA (TE) buffer at a final concentration of $1 \mu \mathrm{g} / \mu \mathrm{L}$. 
Recombinational cloning reactions were carried out by introducing $1 \mu \mathrm{g}$ of donor fragment, $1 \mu \mathrm{g}$ of each linker, and $100 \mathrm{ng}$ of $A f l \mathrm{II}$ linearized YRpETE acceptor (linearizing restriction site locations depicted in Figure 2) into $S$. cerevisiae strain JY128 (ura3-52, trp1$\Delta 63$ ) by electroporation (8). The reaction mixture was subsequently plated on trp- synthetic complete medium fortified with 5-FOA. Recombinational cloning reactions were performed in triplicate, yielding an average of $200 \pm 11$ yeast transformants per reaction. Yeast transformant plasmid DNA isolation (Reference 9, modified by the addition of chloroform extraction prior to ethanol precipitation) was carried out 3-5 days later and used to transform competent Escherichia coli (note that one half of the precipitated DNA was used per $E$. coli transformation). Restriction fragment banding patterns of plasmid DNA isolated from $50 \mathrm{E}$. coli transformant lines revealed that all harbored recombinant plasmids possessing the predicted banding patterns. Dideoxy sequencing of three recombinant plasmids within the regions of PCR-generated homology was performed (sequencing reactions performed at and results obtained from Norgen Biotek, St. Catharines, ON, Canada). A single thymine base deletion was detected within the PCRgenerated regions of homology in one of the three plasmids analyzed. In addition, the integrity of the EGFP reporter gene was maintained throughout recombinational cloning, as evidenced by GFP fluorescence (visualized under $488 \mathrm{~nm}$ wavelengths) of HEK 293 cells (CRL1573; ATCC, Manassas, VA, USA) (10) transfected with $\mathrm{CsCl}$ density gradient centrifugation purified recombinant plasmid (using ExGen ${ }^{\mathrm{TM}} 500$ In Vitro Transfection Reagent; MBI Fermentas, Burlington, ON, Canada).

In order to further demonstrate the utility of URA3 counter-selection during in vivo plasmid construction, partially digested YRpETE acceptor was utilized as substrate during in vivo plasmid construction. It has been demonstrated that partially digested acceptor can be utilized as substrate for in vivo plasmid construction, provided a suitable selection system is available to decrease screening workload (3). The YRpETE acceptor contains three
SacII recognition sites, only one of which [labeled $\mathrm{SacII}(358)$ in Figure 2 ] is in a suitable location to facilitate recombinational cloning with the linkers described in Figure 2. SacII partial digests of YRpETE were carried out as described previously (11). YRpETE acceptor plasmid, cut only once by SacII, was purified from agarose gels for use in recombinational cloning reactions. When purified single-cut SacII partial digests of YRpETE were substituted for AflII-digested YRpETE during recombinational cloning reactions, an average of $50 \pm 9$ yeast transformants growing on trp ${ }^{-}$synthetic complete medium fortified with 5-FOA was observed. All plasmids analyzed from these transformants possessed the predicted restriction fragment banding patterns $(20 / 20$, as determined by isolation of yeast plasmid DNA, E. coli transformation, and restriction enzyme fragment banding pattern analysis).

Very high proportions of background yeast transformants accompanied similar recombinational cloning reactions without URA3 counter-selection. When AflII-digested YRpXC38 acceptor was utilized under identical recombinational cloning reaction conditions without URA3/5-FOA counterselection, restriction fragment banding patterns of plasmid DNA isolated from 70 ampicillin-resistant bacterial colonies revealed that only three harbored the recombinant plasmid (the remainder contained regenerated acceptor plasmid). Similar recombinational cloning experiments using single-cut SacII partial digests of YRpXC38 yielded only regenerated acceptor plasmid in 100 ampicillin-resistant E. coli transformants screened by restriction fragment banding pattern analysis.

The restriction sites necessary for incorporation of both the yeast selectable markers and ARS were present in positions convenient for the construction of the acceptor plasmids described herein. This may not be the case for other cloning vector systems. The TRPI/ARSI and URA 3 fragments utilized in this report are sufficiently short that they can be amplified by PCR and subsequently utilized for two-fragment recombinational cloning reactions (4). Such a strategy has been undertaken, and we have yet to observe any recombinants other than those of the expected conformation (as examined by restriction fragment banding pattern analysis, unpublished data). Any integration events or PCR/recombination-induced mutations disrupting the proper function of the markers or replication sequences appear to be effectively filtered out by the selective pressure of plating on the appropriate yeast selective medium and recovery of the recombinant plasmid by transformation into $E$. coli.

Counter-selection facilitated recombinational cloning is capable of generating multiple plasmid constructs with similar backbone DNA sequences in a short time frame. The convenient zero background selection system allows the isolation of recombinant plasmid from a recombinational cloning reaction without the need for time-consuming replica plating. More important than its ability to merely decrease screening workload, this counter-selection technique eliminates problematic background yeast transformants harboring regenerated acceptor plasmid. The elimination of background yeast transformants allows the use of partially digested acceptor as substrate for recombinational cloning, thus increasing the number of useful restriction sites available to the researcher (a common dilemma when manipulating large plasmid systems). Counter-selection facilitated recombinational cloning, therefore, provides an alternative to the generation of novel restriction sites by site-directed mutagenesis or the insertion of multiple cloning sites and should facilitate research involving plasmid systems that are typically refractory to manipulation by traditional in vitro techniques.

\section{ACKNOWLEDGMENTS}

The authors wish to acknowledge the National Sciences and Engineering Research Council of Canada for their financial support.

\section{REFERENCES}

1.Gunyuzlu, P.L., G.F. Hollis, and J.H. Toyn. 2001. Plasmid construction by linker-assisted homologous recombination in yeast. BioTechniques 31:1250-1252. 
2.Ma, H., S. Kunes, P.J. Schatz, and D. Botstein. 1987. Plasmid construction by homologous recombination in yeast. Gene 58:201-216

3.Miletti, K.E. and M.J. Leibowitz. 2000 Construction of large plasmid lacking linearizing single restriction sites by simultaneous plasmid shuffling in yeast. Yeast 16:15271534.

4.Raymond, C.K., T.A. Pownder, and S.L. Sexson. 1999. General method for plasmid construction using homologous recombination. BioTechniques 26:134-141.

5.Tschumper, G. and J. Carbon. 1980. Sequence of a yeast DNA fragment containing a chromosomal replicator and the TRP1 gene. Gene 10:157-166.

6.Bautista, D.S., M. Hitt, J. McGrory, and F.L. Graham. 1991. Isolation and characterization of insertion mutants in E1A of adenovirus type 5. Virology 182:578-596.

7.Boeke, J.D., L. LaCroute, and G.R. Fink. 1984. A positive selection for mutants lacing orotidine-5-phosphate decarboxylase activity in yeast: 5-fluoro-orotic acid resistance. Mol. Gen. Genet. 197:345-346.

8.Becker, D.M. and L. Guarente. 1991. High efficiency transformation of yeast by electroporation. Methods Enzymol. 194:182-187.

9.Hoffman, C.S. and F. Winston. 1987. A ten-minute DNA preparation from yeast efficiently releases autonomous plasmids for transformation of Escherichia coli. Gene 57: 267-272.

10.Graham, F.L., J. Smiley, W.C. Russell, and R. Narin. 1977. Characteristics of a human cell line transformed by DNA from human adenovirus type 5. J. Gen. Virol. 36:59-72.

11.Parker, R.C., R.M. Watson, and J. Vinograd. 1977. Mapping closed circular DNAs by cleavage with restriction endonucleases and calibration by agarose gel electrophoresis. Proc. Natl. Acad. Sci. USA 74:851-855.

Received 16 December 2002; accepted 8 July 2003

Address correspondence to Peter Robert Anderson, University of Guelph, Department of Molecular Biology and Genetics, Guelph, ON, N1G 2W1, Canada. e-mail: panderso@uoguelph.ca

\section{A/T-rich inverted DNA repeats are destabilized by chaotrope-containing buffer during purification using silica gel membrane technology}

\author{
Martin Převorovský and František Půta \\ Charles University, Prague, Czech Republic
}

BioTechniques 35:698-702 (October 2003)
The recovery of DNA from agarose gel is a frequently used step in nearly all gene engineering procedures. Various methods have been developed for this purpose, and the use of silica gel or glass matrices seem to be the most efficient ones. Here we describe the difficulties encountered when purifying DNA fragments consisting of inverted repeat sequences.

In our study, we wanted to determine the knock-down phenotype of several novel Dictyostelium discoideum genes by using RNA interference (RNAi) (1), which is the method of choice when assaying gene function both in genetically well established and in genetically less well-characterized organisms (2). The RNAi method that we employed consisted of the introduction of doublestranded RNA (dsRNA) molecules (usually several hundred base pairs long) that are processed into the effector short interfering RNAs (siRNAs; Reference 3), resulting in sequence-specific mRNA degradation $(4,5)$.

Using the sequences of our candidate genes, we constructed an inverted repeat targeting cassette (RNAi cassette) for each gene, which should produce a hairpin RNA upon transcription. Figure 1 depicts the RNAi cassette construction. The identity of the constructed RNAi cassettes was verified by restriction analysis; Table 1 sum-

To reclone the prepared RNAi cassettes from the cloning vector into the expression vector, we cut the RNAi cassettes using BamHI, separated them from the vector DNA by electrophoresis on $1 \%$ agarose gel, and purified them by gel purification using the QIAquick ${ }^{\mathrm{TM}}$ Gel Extraction Kit (Qiamarizes their properties.

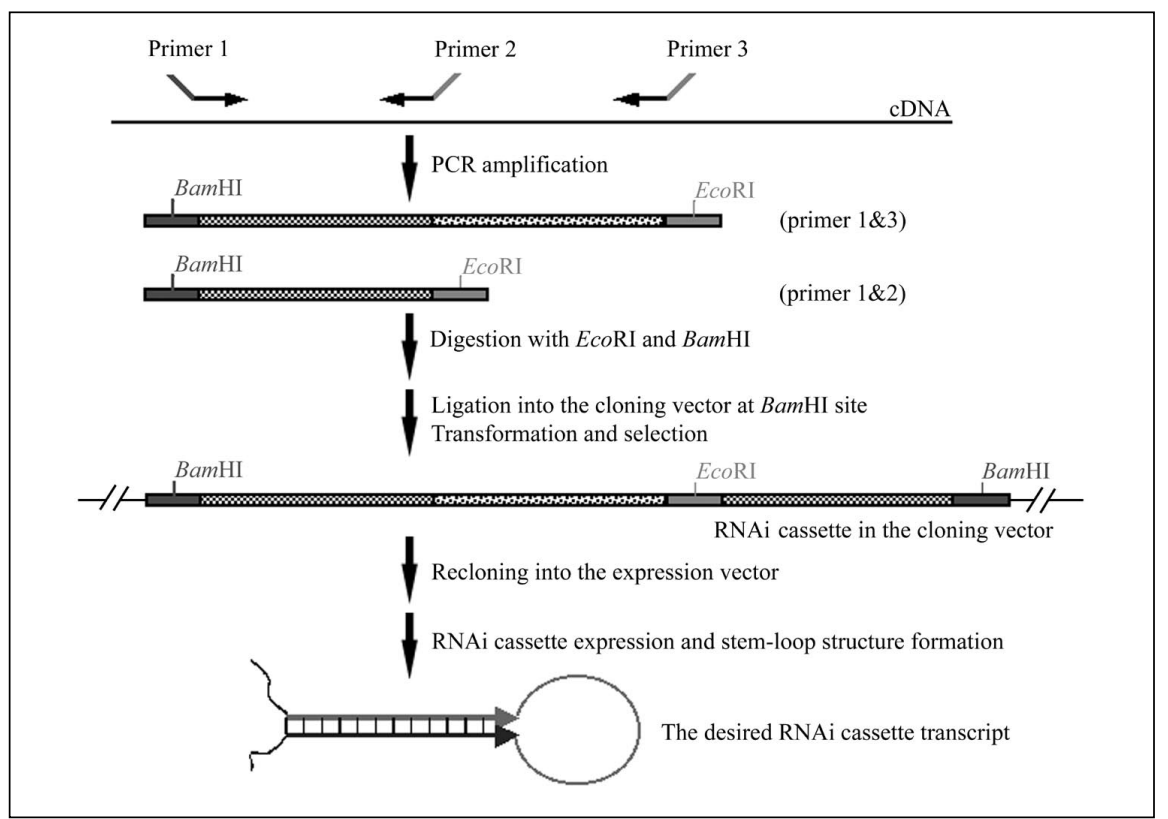

Figure 1. The RNA interference (RNAi) cassette construction procedure and transcribed RNA structure. 